\title{
Intrahepatic Cholangiocarcinoma in a Cirrhotic Patient without Upstream Biliary Dilatation
}

\author{
Guan Huang ${ }^{1 *}$, Safwat Girgis ${ }^{2}$, Gavin Low ${ }^{1}$ \\ ${ }^{1}$ Department of Radiology \& Diagnostic Imaging, University of Alberta Hospital, Canada \\ ${ }^{2}$ Department of Laboratory Medicine \& Pathology, University of Alberta Hospital, Canada
}

Received: December 07, 2015; Accepted: March 15, 2016; Published: 17 May, 2016

*Corresponding author: Guan Huang, Department of Radiology and Diagnostic Imaging, University of Alberta Hospital, 2A2.41 WMC, 8440-112 Street, Edmonton, AB T6G 2B7 Canada, Tel: 1780407 6810; Fax: 1780407 6176; E-mail: guanhuang@gmail.com

\begin{abstract}
Intrahepatic cholangiocarcinoma (IHC) is a biliary tumor that arises from the intrahepatic bile ducts. It is the second most common primary hepatic malignancy after hepatocellular carcinoma (HCC), with higher prevalence in patients with cirrhosis and Hepatitis B and C. On imaging, IHC typically demonstrates progressive centripetal progression and significant delayed enhancement with dilatation of the bile ducts upstream from the tumor. We report a case of IHC in a cirrhotic patient which exhibited delayed enhancement but no evidence of upstream ductal dilatation. Due to differences between IHC and HCC in prognosis and treatment options, IHC should be considered in a cirrhotic patient when the hepatic tumor demonstrates delayed centripetal enhancement on CT and/or MR, even in the absence of upstream biliary dilatation.
\end{abstract}

Keywords: Intrahepatic cholangiocarcinoma; Intrahepatic ductal dilatation; Hepatocellular carcinoma

\section{Introduction}

Intrahepatic cholangiocarcinoma (IHC) is a biliary tumor that arises from the Intrahepatic bile ducts [1]. It is the second most common (15\%) primary hepatic malignancy and is associated with an increasing worldwide incidence over the last several decades [2-4]. Risk factors for IHC include liver flukes, recurrent pyogenic cholangitis, primary sclerosing cholangitis, viral infections including HIV, HBV and HCV, and cirrhosis [5]. IHCs are classified into three categories based on the morphologic growth pattern: mass forming, Periductal infiltrating, and Intraductal growing [6,7]. Mass forming IHC is the most common type and is usually a large well-defined tumor with lobulated margin; Periductal infiltrating IHC grows along bile ducts hence demonstrates a branch-like pattern; Intraductal growing IHC spreads along the mucosal surface and gives rise to multiple small, polypoid masses along bile ducts [4]. Mass forming IHC typically results in upstream biliary dilatation which provides an important clue to diagnosing this disease entity on imaging. However, when biliary dilatation is absent, mass forming IHC can be difficult to differentiate from Hepatocellular Carcinoma (HCC), especially in patients with underlying cirrhosis.

\section{Case Report}

An 84-year-old male with known alcohol-related cirrhosis was admitted to hospital for generalized weakness and shortness of breath on exertion. He was found to have elevated liver enzymes including an ALP of $471 \mathrm{U} / \mathrm{L}$, ALT of $55 \mathrm{U} / \mathrm{L}$, bilirubin of $44 \mu \mathrm{mol} / \mathrm{L}$ and GGT of $954 \mathrm{U} / \mathrm{L}$. His serology for hepatitis A, hepatitis $B$, and hepatitis $C$ was non-reactive. A contrast-enhanced CT showed a $10 \mathrm{~cm}$ infiltrative mass in the cirrhotic liver within the right lobe. The right portal vein was occluded as a result of direct invasion by the mass. On MRI Figure 1, the mass showed T1 hypo intensity, heterogeneous T2 hyper intensity and restricted diffusion. Following intravenous gadolinium administration, the mass was found to be predominantly hypovascular on the arterial phase, and exhibited progressive centripetal progression from the portal phase into the 5-minute delayed phase. No intrahepatic biliary dilatation, capsular retraction or lobar atrophy was evident. Multiple small lesions were visualized throughout the remainder of the liver (not shown) these exhibited similar signal characteristics with the mass and were suspicious for satellite deposits.

The main differential diagnoses in this cirrhotic patient were Hepatocellular Carcinoma (HCC) or Intrahepatic Cholangiocarcinoma (IHC). Given the diagnostic uncertainty, an ultrasound guided biopsy of the mass was performed. Histologic analysis showed moderate to poorly differentiated adenocarcinoma while immunohistochemistry was positive for CK7 and negative for HEP PAR-1, in keeping with a primary pancreaticobiliary tumor such as IHC Figure 2.

\section{Discussion}

A meta-analysis by Palmer and Patel on risk factors for IHC showed that cirrhosis conferred an odds ratio (OR) of 22.9 (95 \% CI 18.2-28.8), hepatitis B 5.1 (2.9-9) and hepatitis C 4.8 (2.4-9.7) [8]. Capsular retraction may be seen adjacent to the tumor; satellite nodules in the remainder of the liver are common at presentation $[9,10]$. Typically, the bile ducts upstream of the tumor are dilated secondary to obstruction with thickening and enhancement of the ductal lining [11]. In longstanding cases of 

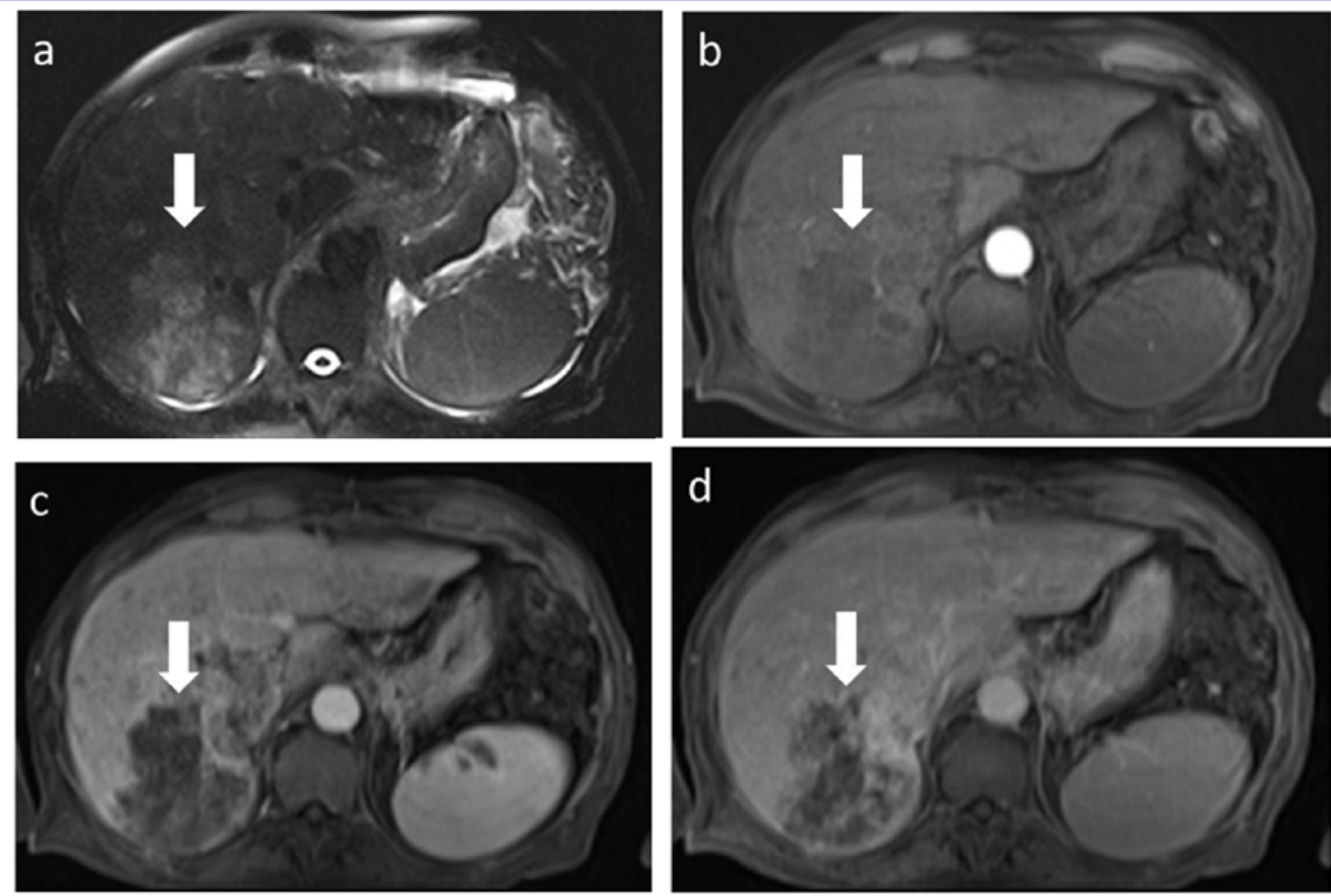

Figure 1: Axial MR images of the cirrhotic liver. (a) the infiltrative mass (arrows) in the right lobe shows heterogeneous high signal intensity on the T2-weighted image; gadolinium-enhanced T1-weighted images show that the mass (arrows) is predominately hypovascular on the arterial phase (b), slightly more enhancing on the portal phase (c) and exhibits marked centripetal fill on the delayed phase (d).
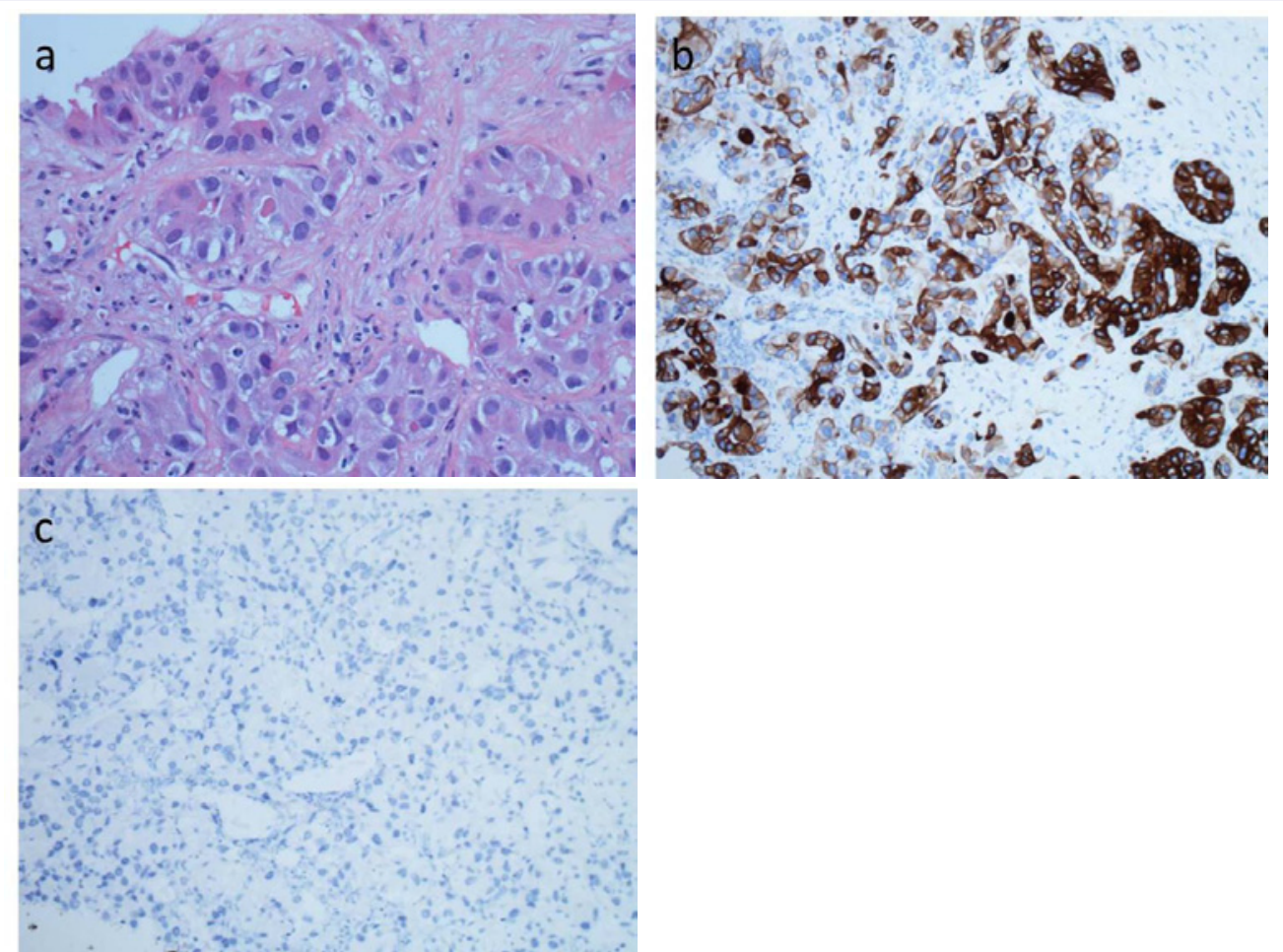

Figure 2: (a) Liver biopsy revealed malignant glands embedded in desmoplastic stroma on routine H\&E stain. Immunochemistry was positive for CK7 (b) and negative for HEP PAR-1 (c). 
ductal obstruction, the involved segment of the liver may show lobar atrophy [12]. On dynamic contrast-enhanced CT or MRI, IHC characteristically demonstrates progressive centripetal progression which is most noticeable on the delayed phase, a feature attributed to dense fibrosis within the tumor [12]. However, delayed tumor enhancement is not an entirely specific feature for IHC as it also recognized in adenocarcinoma liver metastases and confluent hepatic fibrosis $[13,14]$.

HCC is the most common primary malignancy in cirrhosis. The mass did not demonstrate characteristic imaging features for HCC such as avid arterial enhancement and portal or delayed phase washout [15]. On the contrary, the progressive centripetal enhancement pattern was suspicious for IHC. Studies have suggested that IHC may also show peripheral arterial enhancement although the mass in this case was hypovascular on the arterial phase [10].

As it often presents late in the clinical course, IHC carries a poor prognosis with only a $30 \% 5$-year survival. $60-70 \%$ of IHCs are surgically unrespectable at presentation. Furthermore, recurrence rates following potentially curative surgery is high at $50-60 \%$ and the median disease-free survival is only 24 months [16-18]. Liver transplantation is usually contraindicated due to high recurrence rates while the efficacy of locoregional therapies remains inconclusive [16-18]. Palliative therapies such as biliary stenting provide symptomatic relief.

\section{Conclusion}

In summary, IHC should be considered in a cirrhotic patient when the hepatic tumor demonstrates delayed centripetal enhancement on CT and/or MR, even in the absence of upstream biliary dilatation. Biopsy should be performed in equivocal cases as prognosis and treatment options differ between IHC and HCC. Radiologists play a critical role in diagnosis. As per the guidelines of the American Association for the Study of Liver Diseases (AASLD) and the European Association for the Study of the Liver (EASL), a lesion $>1 \mathrm{~cm}$ in a cirrhotic liver that shows arterial enhancement and portal or delayed washout can be diagnosed and treated as HCC without the need for confirmatory biopsy $[15,19]$. Therefore, if IHC is misdiagnosed as HCC radiologically, these cases are likely to be labeled as 'proven $H_{C C}$ ' clinically so run the risk of being managed inappropriately. Conversely, delayed phase imaging should be performed in suspected cases of IHC as it may be the only phase that demonstrates the characteristic enhancement of this type of tumor.

\section{References}

1. Soyer P, Bluemke DA, Reichle R, Calhoun PS, Bliss DF, Scherrer A, et al. Imaging of intrahepatic cholangiocarcinoma: 1. Peripheral cholangiocarcinoma. AJR Am J Roentgenology. 1995; 165: 1427-1431.

2. Khan SA, Thomas HC, Davidson BR, Taylor-Robinson SD. Cholangiocarcinoma. Lancet. 2005; 366: 1303-1314.

3. Lazaridis KN, Gores GJ. Cholangiocarcinoma. Gastroenterology. 2005; 128: $1655-1667$

4. Menias CO, Surabhi VR, Prasad SR, Wang HL, Narra VR, Chintapalli KN
Mimics of cholangiocarcinoma: spectrum of disease. Radiographics : a review publication of the Radiological Society of North America, Inc. 2008; 28:1115-1129.

5. Roth CG, Mitchell DG. Hepatocellular carcinoma and other hepatic malignancies: MR imaging: Radiologic clinics of North America. 2014: 52: 683-707.

6. Maetani $\mathrm{Y}$, Itoh $\mathrm{K}$, Watanabe $\mathrm{C}$, et al. MR imaging of intrahepatic cholangiocarcinoma with pathologic correlation. AJR Am J Roentgenology. 2001; 176: 1499-1507.

7. Lim JH. Cholangiocarcinoma: morphologic classification according to growth pattern and imaging findings. AJR Am J Roentgenology. 2003; 181: 819-827.

8. Palmer WC, Patel T. Are common factors involved in the pathogenesis of primary liver cancers? A meta-analysis of risk factors for intrahepatic cholangiocarcinoma. J hepatology. 2012; 57: 69-76.

9. Vanderveen KA, Hussain HK. Magnetic Resonance Imaging of cholangiocarcinoma. Cancer imaging: the official publication of the International Cancer Imaging Society. 2004; 4: 104-115.

10. Kang Y, Lee JM, Kim SH, Han JK, Choi BI. Intrahepatic mass-forming cholangiocarcinoma: enhancement patterns on gadoxetic acidenhanced MR images. Radiology. 2012; 264: 751-760.

11. Apisarnthanarak P, Pansri C, Maungsomboon K, Thamtorawat S. The CT appearances for differentiating of peripheral, massforming cholangiocarcinoma and liver meatastases from colorectal adenocarcinoma: J Med Assoc Thai = Chotmaihet thangphaet. 2014; 97: 415-422.

12. Chung YE, Kim MJ, Park YN, Jin-Young Choi, Ju Yeon Pyo, Young Chul Kim, et al. Varying appearances of cholangiocarcinoma: radiologicpathologic correlation. Radiographics. 2009; 29: 683-700.

13. Kelekis NL, Makri E, Vassiou A, Patsiaoura K, Spiridakis M, Dalekos GN. Confluent hepatic fibrosis as the presenting imaging sign in nonadvanced alcoholic cirrhosis. Clin Imaging 2004; 28: 124-127.

14. Gabata T, Matsui O, Kadoya M, Yoshikawa J, Ueda K, Kawamori Y, et al. Delayed MR imaging of the liver: correlation of delayed enhancement of hepatic tumors and pathologic appearance. Abdom Imaging 1998; 23: 309-313.

15. Josep ML , Michel D. European Association for the Study of the Liver, European Organisation for Research and Treatment Of Cancer. EASLEORTC clinical practice guidelines: management of hepatocellular carcinoma. J Hepatol. 2012; 56: 908-943.

16. Hu XX, Yan LN. Retrospective analysis of prognostic factors after liver transplantation for intrahepatic cholangiocarcinoma in China: a single-center experience. Hepatogastroenterology. 2011; 58: 12551259.

17. Singal A, Welling TH, Marrero JA. Role of liver transplantation in the treatment of cholangiocarcinoma. Expert review of anticancer therapy. 2009; 9: 491-502.

18. Guglielmi A, Ruzzenente A, Campagnaro T, Pachera S, Valdegamberi $A$, Nicoli $\mathrm{P}$, et al. Intrahepatic cholangiocarcinoma: prognostic factors after surgical resection. World J Surg. 2009; 33: 1247-1254.

19. Bruix J, Sherman M, American Association for the Study of Liver Disease. Management of hepatocellular carcinoma: an update. Hepatology. 2011; 53: 1020-1022. 